\title{
Augmented Reality (AR) for utility infrastructure: An experiential development workflow
}

\author{
Dr. Poorang Piroozfar ${ }^{1,2,3,4}$, Mr. Alex Judd ${ }^{1,3}$, Mr. Simon Boseley ${ }^{3}$, Mr. Amer Essa ${ }^{3}$, \\ Dr. Eric R. P. Farr ${ }^{2,3,4}$ \\ ${ }^{1}$ School of Environment and Technology, University of Brighton, Brighton, BN2 4GJ, UK \\ ${ }^{2}$ Digital Construction Lab, University of Brighton, Brighton, BN2 4GJ, UK \\ ${ }^{3}$ MAVRiC Research and Enterprise Group, Shoreham-by-Sea, BN43 6AX, UK \\ ${ }^{4}$ NONAMES Design Research and Studies, 1249 F Street, San Diego, CA 92101, USA \\ A.E.Piroozfar@brighton.ac.uk
}

\begin{abstract}
The process and product development phase of the research instrument for experiential action research is crucial in the success of the research. Due to time, space and resource limitations, fewer studies have concentrated on this development process. In this respect, research on Augmented Reality (AR) in the architecture, engineering and construction (AEC) industry is no exception. This is more evident in subsurface, urban utilities and infrastructure sector. Furthermore, a limited number of studies on AR/VR have utilized mobile devices as their enabling technologies. This paper sets out to contribute to the state-of-the-art in AR research for urban utilities and infrastructure by outlining a generic procedural workflow to be used for designing AR experiments for experiential research in this area. Given the fact that workflow development research in AR is still limited, this research presents a unique contribution in this area to date.
\end{abstract}

Keywords: Augmented Reality, Construction Industry, Handheld Devices, ICT Application, Infrastructure, Urban Utilities.

\section{Introduction}

The process and product development phase of the research instrument for experiential action research is crucial in the success of the research because it (re)defines the researchersubject relationship and promotes the role and facilitates engagement of the subject as a coresearcher in such studies. Therefore, the importance of the design of such experiments as the backbone of the research instrument remains undebatable in experiential studies. Due to time, space and resource limitations, fewer studies have concentrated on this development process. In this respect, research on Augmented Reality (AR) and Virtual Reality (VR) in the architecture, engineering and construction (AEC) industry is no exception. A limited number of studies on AR/VR have utilized mobile devices as their 'Enabling Technologies'. In the case of AR/VR research for urban utilities and infrastructure, the impact of the choice of device on health and safety (H\&S), as well as legal and liability concerns are issues that have driven the choice of device away from HMDs towards handheld devices. This makes it difficult to generalize the knowledge claims of such studies as they remain context-specific with limited scope for triangulation of findings. Therefore, this paper sets out to contribute to the state-of-the-art in AR research for urban utilities and infrastructure by outlining a generic procedural workflow to be used for designing AR experiments for experiential 
research in this area. Literature review on AR and its associated aspects and areas is presented first. The paper then carries on with the AR experiment, developed to be applicable to a variety of mobile devices available on the current market. Depending on the devices used (and their respective operating systems), minor adjustments to the experiment might be inevitable. Given the fact that workflow development research in AR is still limited, this research presents a unique contribution in this area to date.

\section{Literature Review}

In comparison with VR, AR is relatively new and as such its definitions are still subject to transformation. The most widely agreed definition of AR seems to be what Milgram and Kishino [1] have proposed, where they place AR on a spectrum between physical reality and virtual reality, taxonomizing it as a form of "Mixed Reality". However, the term is now more likely to refer to any case in which an otherwise real environment is "augmented" by means of virtual or computer graphic objects.

\subsection{Data Availability}

Utility asset data availability determines the approach to, precision and effectiveness of the AR instrument devised to assist in upkeep, maintenance and repair of the utility network. The current status of utility data is in need of some improvements. Previous researchers highlighted the lack of digital formats [2] and inaccuracy of as-built information [3]. The need for a shared geospatial platform is suggested to be key to handheld AR applications [4], especially with reference to mobile market hardware developments [5]. This has been suggested to the extent that utility data will eventually become as accessible as Google ${ }^{\mathrm{TM}}$ [6], where asset owners and in particular local authorities have been encouraged to make their data more accessible to enable safe excavation [7]. Doing so also enables AR technologies to link with large quantities of information, hosted by BIM enabled platforms, streamlining and simplifying its application [8]. Other countries such as Singapore have begun to make their infrastructure data more accessible, where various benefits to procurement of such projects are being realized [9] with some direct benefits for quality assurance as well as facilitating visualization methods. Although with increased use of GPS technologies, data collection and storage are beginning to merge [3], interoperability and encapsulation of non-asset data remains a challenge and may affect excavation and space planning practices [10].

\subsection{Data Accuracy}

With regards to data and information quality, the accuracy of the source data is a matter of concern in almost every research on subsurface utilities in conjunction with AR [2, 3, 11, 12], where the role that experts can play in public safety [12] and complexity [13] have been highlighted. PAS128:2014 [14] recommends ground penetrating radars (GPR) high accuracy of $150 \mathrm{~mm}$ which has been adopted by some researchers [15] with others suggesting $300 \mathrm{~mm}$ $[4,16]$ or even $500 \mathrm{~mm}$ [11]. Other elements associated with accuracy relate to capturing, visualization and positioning. For instance, GPR limitation in capturing data of dead power cables; low pressure gas and water pipes [7]; and new plastic pipes [2] have been discussed in previous research. Technology development will allow for more reliable data capture such as pit photogrammetry and gyroscopic mapping with accuracy well in succession of $150 \mathrm{~mm}$ [9], while utilizing a variety of surveying methods has been proposed to enable accurate data 
capture [3, 10]. Human errors and surveyors' skill and competence [3] and their ability to locate the pipe on site [17] are however not to be undermined.

\subsection{Model Content}

The requirements of augmented utility model contents have been broadly discussed, highlighting the importance of factors such as: size and shape [3]; color [16]; and transparency [18]. While Talmaki et al. [3] advocate that the shape of utilities should differ as per cross-section type, review of other research suggests, to the contrary, that modelling objects should be kept at a lower level of detail [19]. Regardless, it is important that objects are projected to scale and have a coherent colour coding schema [20]. In order to negate the negative effects of occlusion, a semi-transparent visualization can be used [18]. Filtering the data [4] and simplifying the visualization can avoid misperceptions [19]. Therefore, a suitable working range must be implemented, for which $5 \mathrm{~m}$ has been suggested $[17,21]$. It is suggested that as well as the utility objects, the models also need to consider scene composition plans [16], or a rendered 3D terrain [3]. Others do not concur with this opinion pointing at cost implications [22] or increasing chance of clash with real-world features [12]. Communicating the uncertainties associated with visualization accuracy was discussed as an essential requirement for operators [3], which could cause model over-complication. One suggested solution is meta-information labelling [4], permitting informed field decisions [3] and allowing for rapid cloud-enabled access to data [6]. Previous research also highlights requirements for geophysical meta-data to inform excavation techniques [13]. Others have found that informing field workers of extra tasks and tools provides little benefit [4].

\section{$2.4 \quad$ Platform}

A robust platform is essential for hosting the visualization. AR is often hosted on a mobile or a wearable device. However, due to dynamic and high-risk environment of construction sites, mobile technologies are favoured [6]; with benefits highlighted as portability, cost and availability [8], while their ability to convey more detailed information has been disputed by others [6]. However, there are some downfalls including their inability to be hands-free [20] and their apparent depth perception issues [16]. The platform also needs to be ergonomic [19] while daylight affecting the user's experience has also been widely discussed [4, 17, 21], suggesting that methods to eradicate glare and reflection need to be considered. It is suggested that a laptop or a screen can resolve these problems. However, they would require two hands. Therefore, they need to be mounted and screen interactivity should be kept at minimum [4]. Stable localization technologies need to be implemented for higher accuracy [8]. Registration is still highlighted as a shortcoming for AR [12]. To achieve good registration, some propose using a simultaneous localization and mapping (SLAM) system which will enable continuous data transmission in the instance of a sensor failure [8].

\subsection{Procurement}

Even with highly coherent and accurate augmentations, its application needs to be justified to ensure correct use. Therefore, the procurement of the system has been investigated during planning, analysis and excavation stages while analysing its implications on people and site technology. Insufficiently planned construction work can be hazardous [19] especially where the work sequence is counterintuitive. The UK government recognizes this in urban utility sector and to respond to this need, produced PAS 128:2014 (Specification for underground utility detection, verification and location) in 2014. Previous research raises awareness of an 
evident gap between construction practices and mapping disciplines [3, 8, 10]. AR could close this gap by allowing fieldworkers to connect with remote colleagues $[5,20]$ either through screen sharing or through attribute editing/redlining. There is some debate as to the responsibility of producing 3D geometric assets on-site, where Schall et al. [16] propose model interaction and allowing on-site digital asset production and changes to meta-data will facilitate this. The benefit of on-site model control is in the inclusion of adjustments to existing utilities [3], often not picked up in the back office. A concern of modern-day utility excavation practices is the process of imagination that surmounts from the lack of persistent visual guidance [3] and the undetermined distance of the excavator bucket to the pipe crown. Behzadan et al. [11] suggest the use of real time forward kinematic algorithms to accurately calculate this distance as well as a combination of audio-visual alert systems to the operator, while Talmaki et al. [3] suggest proximity analysis. A criticism of such an AR system is that it may give the operator a false sense of accuracy [2] giving the impression that reducing these safety nets due to advanced technologies will result in the same H\&S levels. LSBUD [7] suggests that $44 \%$ of works in the UK take place without a utility search. Previous research suggests that an AR system may improve this statistic through increasing awareness of utilities by excavation teams [6]. However, even if the AR system is robust, safety concerns can occur from personal behaviour and attitude of the AR operators [19]. It is suggested that although, even well-trained workers may have a negligent attitude towards safety, visual literacy skills should be improved to allow effective AR usage [19]. Simultaneous use of the platform by more than one user can ensure safe procurement. Some researchers suggest that interactivity provides a more meaningful overlay visualization [15], facilitating improved performance in users tasks [3] while raising safety concerns [8], thereby suggesting that the excavator operator should have minimal interaction. AR helps contractors with discovery-based learning methods [22], allowing operators to understand how to avoid utility strikes as well as how to deal with a strike if it occurs; essential for modern complex engineering projects [19].

\section{$3 \quad$ Research Design and Methodology}

To carry out experiential or applied research in AR, the initial stage is to develop an experiment tool. After the preliminaries were carried out an experiment was designed to ensure objectives would be achieved, fulfilling the research questions. The aim of this paper is to expand on the development process of the experiment. The experiential nature of the research enquiry required that the experiment be designed, accounting for the research participants where separation of the researcher's and the subject's roles dissolves to enable those involved to become co-researchers and co-subjects, to devise, manage and draw conclusions from the research, but also to undergo the experiences and perform the actions that are being researched [23]. Therefore ease of use, practicality, interactivity, and active engagement were the most important criteria in the research design, among more common factors such as replicability, validity, reliability, reproducibility of the instrument and the process of data enquiry and analysis. In doing so, special attention was given to the value of human experience; focusing on the wholeness of experience; searching for meanings and essences of experience; obtaining descriptions of experience through first-person account; regarding the experiential data as imperative; formulating questions and problems that reflect the interest, involvement, and personal commitment of the researcher; and last but not least, viewing experience and behavior as integrated and inseparable discourses as indicated by Piroozfar et al. (2018). 


\section{Experiment Development}

\subsection{Model Development}

A 3D model representing urban utilities assets was required and accordingly devised based on aspects of the literature review and as specified in PAS128 Quality Level B. Utility survey data was optimized in Autodesk Civil 3D using the pipe network features. Civil 3D allows for pipes and structures to be generated from this data, however to save time the plug-in "PipesToolBox" was utilised to batch-swap the imported objects into their correct layers and networks. These were then exported as AutoCAD 3D Objects for integration with the

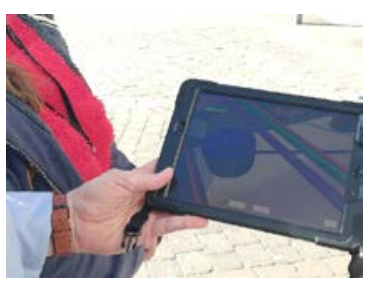

Figure 1: Developed app Unity platform, where the development of the application could be initiated.

\subsection{Application Development}

In developing the experiment, an investigation was first carried out to establish the most suitable solution regarding AR for urban utilities. The initial solution was to identify a multiplatform application (including Android and iOS) to display GPS-located 3D AR models. Previously, an application called 'LayAR' was used to this effect [24], however as of 2019, LayAR no longer supports this level of functionality. The solution, therefore was to instead develop an in-house standalone application that provides all required features. 'Unity' game engine was chosen to build the app, due to its multi-platform accessibility and support for the desired features, as well as being free to use and frequently updated. Next, several APIs (Application Programming Interface) for AR were trialled; the selected API would serve as the 'AR engine' to provide 3D AR functionality utilizing the mobile device's camera. The APIs tested include: Wikitude, Mapbox, Vuforia, AR Foundation and ARCore. After trialling these APIs, the application was developed with Vuforia with the aim of developing a script to provide GPS functionality. Due to time restraints, it was not possible to develop such functionality in-house and so, third-party plug-ins were explored of which, 'AR+GPS' plug-in was deemed most suitable. Initially, there were software compatibility issues between Vuforia and the GPS plug-in. As a result, GPS was temporarily abandoned as an app feature. At this point, the ARCore API was chosen to replace Vuforia. ARCore allowed for 'surface-tracking' AR (figures 2a and 2b) - using ground surface planes to detect and augment utilities - without GPS functionality. Given that GPS was a desired feature of the application, the AR+GPS plug-in was revisited and trialled with a different API: AR Foundation. This combination worked and so GPS-based AR was possible and with Unity this meant the app could be used on a variety of mobile platforms. With a rudimentary app developed, features were added to improve performance, user experience and feedback. To
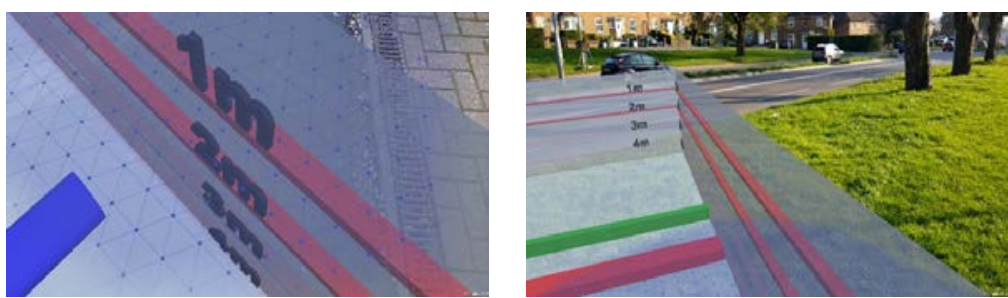

Figure 2a (left) Surface tracking of ground plane using ARCore Grid; and $2 \mathrm{~b}$ (right) SLAM-like grid (represented by scattered magenta dots) 
provide debugging information so that the app could be improved and also serve as a user feature, a compass as well as relative GPS data were built-in. From this we could analyse how accurate the GPS data was. The next step was to include multiple 3D models in the app to represent utilities at different sites/locations. The aim was to have these 3D elements overlaid with GPS synchronized. After trialling this, there were issues with lost performance and overlaying of multiple site models in one instance. To address this, a UI (user interface) system was developed so that only a single model would be shown at any particular time, where the user could switch between different geo-tagged models. The app was modified to display hotspots ${ }^{1}$ indicating site locations based on global positioning (figure 3 ), at each location, where the user could switch to the relevant site model and at the appropriate size (a 20x20 or 50x50 meter trench). The final stage of application development looked into inclusion of layer functionality and

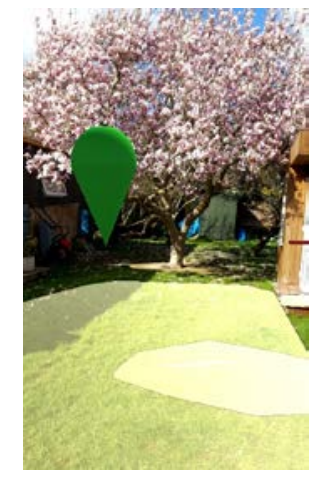

Figure 3: Hotspot indicating site location meta-data of the associated pipework. A separate UI was developed to allow for navigation and enabling the relevant utilities as and when deemed required, including: main supply (e.g. gas, electric, water mains); drainage network; and communication infrastructure (figures 4a and $4 \mathrm{~b}$ ). To enhance the geo-locationing feature of the application, a GLONASS (Global Navigation Satellite System) GPS enhancer device was procured to pair up with the mobile device and help with real-time locationing. This intended feature did not work due to the plug-in limitation in allowing for the GPS enhancer to take over the internal mobile GPS. Further work would be required to explore if the device or the plug-in can be coded or configured in order to take over the device GPS via Bluetooth ${ }^{\mathrm{TM}}$.
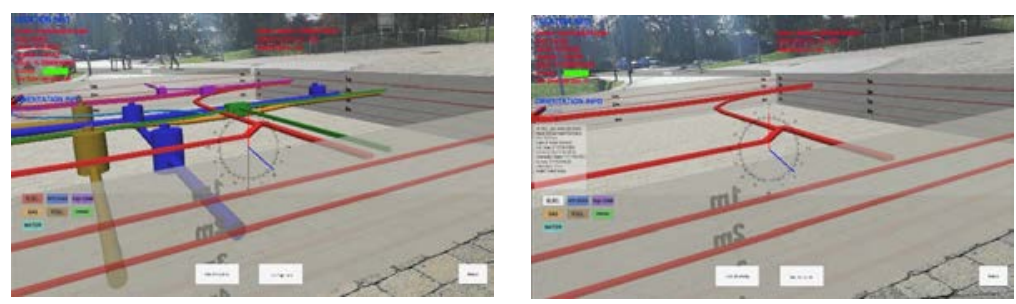

Figure 4a (left) layered view of all augmented utility types and; 4b (right) metadata of the isolated utility type (chosen utility: electricity duct network)

\section{Concluding Comments and Future Research}

Due to new development of affordable, user-friendly and open-source applications, conducting such level of research and development is now possible; what was not even conceivable a decade or so ago. However, this still looks more or less like a 'black box' to many and is worth shedding some lights on. This gap has been indicated in previous experiential research on application of both AR and VR in the AEC industry especially where a user-centered research instrument has been intended. It was noted that in such approaches to action research, no longer does traditional division between the researcher and the subject exists and the participants will be promoted to the role of co-subjects/co-researchers and therefore it is of paramount importance that every measure is taken to improve their

\footnotetext{
${ }^{1}$ This is an AR/VR development technical term and differs from what it may denote as mobile network
} 
engagement, enhance their experience, protect their H\&S and other individual or social interests. To help bridge this gap, a series of experiments has been developed with ease of use, applicability and fitness for purpose of the developed experiment in the center of focus. To serve the specific purpose of this paper, the experiment has been revisited and redesigned to ensure that it stays generic and presents a customizable workflow which can be adopted and adapted to the specifics of similar research in the field. An intensive trial and error exercise was carried out which, although seminal to this development process, was kept to a minimum to avoid discouraging the readers. Most areas of concern were related - directly or indirectly - to software-to-software and/or software-to-hardware interoperability. Therefore, it is advisable to maximize the use of open-source software in case coding was deemed required as the only way forward, if possible at all, to improve on this aspect. The next point worth mentioning is coding skills required. Again with more and more coding languages moving towards Visual Programing Languages (VPLs) protocols, this task has become much more easily manageable with limited to no previous experience required. The next problem was that GPS is not as accurate as needed for the specific purpose of this research. This was expected as GPS can only provide certain levels of accuracy. This however, was still within the accuracy limits for AR application in urban infrastructure and utility research and practice as suggested in previous research, hence the chosen method in this study. To tackle this problem, the use of local positioning systems seems to be the way forward, either on their own or in combination with GPS. However, the practicality of linking the two might be problematic. Another outstanding issue to resolve is altitude and height, where relying merely on GPS data does not suffice and requires an additional layer of sourcing and inclusion of data. The need for meta-data to complement the data visualization was another important requirement which was uniformly picked up in the pilot study and was added to the final prototype. Coupled with meta-data was the capability to annotate which was mentioned as a much-needed capability within the application. This was kept for further development to avoid over-complication of the task flow and process, and also for the fact that it could contribute to data or information overload; what was not limited to annotation and imposed a significant hurdle in many other areas. Therefore, the general advice is to avoid inclusion of any feature or data/information categories unless they are absolutely necessary, reducing the risk of distraction and threat on H\&S. Distraction and H\&S were also highlighted - as in previous research - to be associated with other areas such as the type, the size and the ease of use of the device; its location; the type, time, frequency and pace of the user's interaction with the device; device data update; refresh rate and effectiveness; Wi$\mathrm{Fi}$ and Bluetooth ${ }^{\mathrm{TM}}$ effectiveness; screen brightness and readability in daylight, to name but just a few. One of the other solutions to overcome the problem of data clutter we suggest is to add a 'Section Box' where sections of the visualization - vertical or horizontal - can be cropped from view to speed up the application. Links to GIS databases were also picked up as beneficial aspects to include and improve on. Although many of the above-mentioned areas were concentrated and improved during the several rounds of iteration for application development in this study, there is still more work to be done in those areas, which will set the target for future research in many of those areas.

\section{References}

1. Milgram, P. and F. Kishino, A taxonomy of mixed reality visual displays. IEICE TRANSACTIONS on Information and Systems, 1994. E77-D(12): p. 1321-1329.

2. Roberts, G., et al. The location and positioning of buried pipes and cables in built up areas. in Proceedings of XXIII Fig Congress: Shaping the Change. 2006. Munich, Germany: XXIII FIG Congress. 
3. Talmaki, S., V.R. Kamat, and H. Cai, Geometric modeling of geospatial data for visualization-assisted excavation. Advanced Engineering Informatics 27, 2013: p. 283-298.

4. Schall, G., et al., Handheld augmented reality for underground infrastructure visualization. Personal and ubiquitous computing, 13(4), 2009: p. 281-291.

5. Nicholls, G. and I.S.T. Powertech, Using augmented reality as an extension to utility GIS. IST Powertech journal, 2013: p. 62-64.

6. Fenais, A., et al. Meta-Analysis of Augmented Reality Challenges in the Underground Utility Construction Industry. in Construction Research Congress 2018. 2018. Tempe, Arizona.

7. LSBUD, Digging Up Britain: How Vulnerable is our Utility Infrastructure? 2017, LinesearchbeforeUdig: London, UK.

8. Chi, H.-L., S.-C. Kang, and X. Wang, Research trends and opportunities of augmented reality applications in architecture, engineering, and construction. Automation in Construction, 2013: p. 116-122.

9. Van Son, R., et al., A framework for reliable 3-D underground utility mapping for urban planning International Archives of the Photogrammetry, Remote Sensing \& Spatial Information Sciences., 2018.

10. Yan, J., et al., THREE-DIMENSIONAL DATA MODELLING FOR UNDERGROUND UTILITY NETWORK MAPPING. nternational Archives of the Photogrammetry, Remote Sensing \& Spatial Information Sciences., 2018.

11. Behzadan, A.H., S. Dong, and V.R. Kamat, Augmented reality visualization: A review of civil infrastructure system applications. Advanced Engineering Informatics, 2015: p. 252-267.

12. Côté, S. and A. Mercier. Augmentation of Road Surfaces with Subsurface Utility Model Projections. in IEEE Conference on Virtual Reality and 3D User Interfaces (VR). 2018. Reutlingen, Germany: IEEE.

13. Jung, Y.J., Evaluation of subsurface utility engineering for highway projects: Benefit-cost analysis. Tunnelling and underground space technology, 27(1), 2012: p. 111-122.

14. BSI, PAS128: Specification for underground utility detection, verification and location. 2014, British Standards Institute: London.

15. Junghanns, S., G. Schall, and D. Schmalstieg, Employing location-aware handheld Augmented Reality to assist Utilities Field Personnel. 2008, Springer.

16. Schall, G., S. Zollmann, and G. Reitmayr, Smart Vidente: advances in mobile augmented reality for interactive visualization of underground infrastructure. Personal and ubiquitous computing, 17(7), 2013: p. 1533-1549.

17. Roberts, G.W., et al., The use of augmented reality, GPS and INS for subsurface data visualization. FIG XXII International Congress, 2002: p. 1-12.

18. Côté, S. and G.-V. Antoine. Accurate OnSite Georeferenced Subsurface Utility Model Visualisation. in International Conference on Augmented and Virtual Reality. 2015. Springer, Cham.

19. Li, X., et al., A critical review of virtual and augmented reality (VR/AR) applications in construction safety. Automation in Construction, 2018: p. 150-162.

20. Meehan, B., NJ Utility on Forefront with New Mixed Reality Application, in ESRI. 2017.

21. Côté, S., I. Létourneau, and J. Marcoux-Ouellet. Augmentation of Live Excavation Work for Subsurface Utilities Engineering. in ISMAR International Symposium on Mixed and Augmented Reality. 2014. Munich, Germany: IEEE.

22. Behzadan, A.H. and V.R. Kamat. Interactive Augmented Reality Visualization for Improved Damage Prevention and Maintenance of Underground Infrastructure. in Construction Research Congress 2009: Building a Sustainable Future. 2009.

23. Heron, J., Empirical validity in experiential research. 1982, Guildford: University of Surrey.

24. Piroozfar, P., et al. The application of Augmented Reality (AR) in the Architecture Engineering and Construction (AEC) industry. in The Tenth International Conference on Construction in the 21st Century (CITC-10). 2018. Colombo, Sri Lanka. 